\title{
Uniqueness of q-difference Polynomials of Meromorphic Functions
}

\author{
Keyu Zhang \\ Department of mathematics of \\ Qilu Normal University \\ Jinan, Shandong 250013 P.R.China
}

\begin{abstract}
In this paper, Applying the theory of Nevanlinna, we investigated uniqueness problem of difference polynomial of meromorphic functions and obtained uniqueness theorems of meromorphic functions, which Extended and improved the results of literature[5].
\end{abstract}

Keywords-uniqueness of meromorphic functions; qdifference; share value; small functions

\section{INTRODUCTION AND MAIN RESULTS}

With the development of difference analogue of Nevanlinna theory, many authors paid their attentions to the value distribution of difference polynomials [1-5]. In particular, the difference logarithmic derivative lemma, given by Chiang and Feng [6], Halburd and Korhonen [7], plays an important part in considering the difference analogues of Nevanlinna theory.

In this paper, we assume that reader is familiar with the standard notations and results of Nevanlinna theory, see [8$11]$.

K.Liu, X.L.Liu, T.B.Cao in [12] got the following resuls.

Theorem $\mathbf{A}^{[12]}$ Let $f$ and $g$ be transcendental meromorphic functions of finite order, suppose that $C$ is nonzero constant and $n \in N$. If $n \geq 14 . f^{n} f(z+c)$ and $g^{n} g(z+c)$ share $1 \mathrm{CM}$, then $f \equiv t g$, or $f g=t$, where $t^{n+1}=1$.

Theorem $\mathrm{B}^{[12]}$ Let $f$ and $g$ be transcendental meromorphic functions of finite order, suppose that $C$ is nonzero constant and $n \in N$. If $n \geq 26 . f^{n} f(z+c)$ and $g^{n} g(z+c)$ share $1 \mathrm{IM}$, then $f \equiv t g$, or $f g=t$, where $t^{n+1}=1$.

In this paper, we will investigate the uniqueness of q-difference polynomials and obtain the following theorems.

Theorem 1. If $f(z)$ is a transcendental meromorphic functions of zero order, If $f^{n}(z) \prod_{i=1}^{d} f\left(q_{i} z\right)$ and $g^{n}(z) \prod_{i=1}^{d} g\left(q_{i} z\right) \quad$ share $1, \infty \quad, \mathrm{CM}, \quad n, k, m, d$ are positive integer and $n \geq 4 d+4$, then $f=\operatorname{tg}, t^{n+d}=1$.

Theorem 2. If $f(z)$ is a transcendental entire functions of zero order, If $f^{n}(z)\left(f^{m}(z)-1\right) \prod_{i=1}^{d} f\left(q_{i} z\right) \quad$ and $g^{n}(z)\left(g^{m}(z)-1\right) \prod_{i=1}^{d} g\left(q_{i} z\right)$ share $1 \mathrm{CM}, n, k, d, m$ are positive integer and $n \geq m+5 d$, then $f=t g$, $t^{n+d}=t^{m}=1$.

\section{PRELIMINARY LEMMAS}

Lemma $1^{[8]}$ Let $f$ be a non-constant meromorphic function, $\alpha_{i}(i=1,2,3)$ be small functions with respect to $f$, then $T(r, f) \leq \sum_{i=1}^{3} \bar{N}\left(r, \frac{1}{f-a_{i}}\right)+S(r, f)$

Lemma $2^{[4]}$ Let $f$ be transcendental meromorphic functions of zero order, $q \in C \backslash\{0\}$, then

$$
T(r, f(q z))=T(r, f)+S_{q}(r, f)
$$

Lemma $3^{[4]}$ Let $f$ be transcendental meromorphic functions of zero order, $q \in C \backslash\{0\}$, then

$$
N(r, f(q z))=N(r, f)+S_{q}(r, f)
$$

Lemma $4^{[11]}$ Let $f$ be transcendental meromorphic functions of zero order, $q \in C \backslash\{0\}$, then

$$
m\left(r, \frac{f(q z)}{f(z)}\right)=S_{q}(r, f)
$$


With the same methods of Lemma 2.4 in [12],we can get the following lemma 5.

Lemma 5 Let $f^{n}(z)\left(f^{m}(z)-1\right) \prod_{i=1}^{d} f\left(q_{i} z\right)$.If $f$ be transcendental entire functions of zero order

$$
T(r, F)=(n+m+d) T(r, f)+S_{q}(r, f) .
$$

If $f$ be transcendental meromorphic functions of zero order, then

$$
\begin{aligned}
& T(r, F) \geq(n+m-d) T(r, f)+S_{q}(r, f) . \\
& T(r, F) \leq(n+m+d) T(r, f)+S_{q}(r, f) .
\end{aligned}
$$

\section{PROOF OF THEOREM 1}

Proof of theorem 1. From the conditions of theorem 1, we know $\frac{f^{n}(z) \prod_{i=1}^{d} f\left(q_{i} z\right)-1}{g^{n}(z) \prod_{i=1}^{d} g\left(q_{i} z\right)-1}=c$,

$C$ is nonzero constant,so we rewriting it as

$$
f^{n}(z) \prod_{i=1}^{d} f\left(q_{i} z\right)-1+c=c g^{n}(z) \prod_{i=1}^{d} g\left(q_{i} z\right)
$$

$$
\text { First we let } \quad F=f^{n}(z) \prod_{i=1}^{d} f\left(q_{i} z\right) \text {, }
$$

$$
G=g^{n}(z) \prod_{i=1}^{d} g\left(q_{i} z\right) .
$$

If $c \neq 1$, From (1) and the lemma 1 , we have

$$
\begin{aligned}
& T(r, F) \leq \bar{N}(r, F)+\bar{N}\left(r, \frac{1}{F}\right)+\bar{N}\left(r, \frac{1}{F-1+c}\right) \\
& +S(r, f) \leq(1+d) T(r, f)+(1+d) T(r, f) \\
& +\bar{N}\left(r, \frac{1}{G}\right)+S(r, f) \\
& \leq(2+2 d) T(r, f)+(1+d) T(r, g) \\
& +S(r, f)
\end{aligned}
$$

From the lemma 5, we know

$T(r, F) \geq(n-d) T(r, f)+S_{q}(r, f)$

Combining (2) and (3), we have

$$
\begin{aligned}
(n-3 d-2) T(r, f) & \leq(1+d) T(r, g) \\
& +S_{q}(r, f)+S_{q}(r, g)
\end{aligned}
$$

Applying the same methods of (4),we have

$$
\begin{aligned}
(n-3 d-2) T(r, g) & \leq(1+d) T(r, f) \\
& +S_{q}(r, f)+S_{q}(r, g)
\end{aligned}
$$

Combining (4) and (5), we have

$$
(n-4 d-3)(T(r, f)+T(r, g))
$$

$$
\leq S_{q}(r, f)+S_{q}(r, g)
$$

Which is a contradicts with $n \geq 4 d+4$,

Then $c=1$, from (1), we have

$$
f^{n}(z) \prod_{i=1}^{d} f\left(q_{i} z\right)=g^{n}(z) \prod_{i=1}^{d} g\left(q_{i} z\right) \text {. }
$$

Let $h(z)=\frac{f(z)}{g(z)}$, then we have

$$
h^{n}(z) \prod_{i=1}^{d} h\left(q_{i} z\right)=1 \text {, so } h^{n}(z)=\frac{1}{\prod_{i=1}^{d} h\left(q_{i} z\right)} \text {, }
$$

So

$$
\begin{aligned}
& n T\left(r, h^{n}(z)\right)=T\left(r, \prod_{i=1}^{d} h\left(q_{i} z\right)\right) \\
& =d T(r, h(z))+S_{q}(r, h)
\end{aligned}
$$

Which is a contradicts with $n \geq 4 d+4$, so $h(z)$ is a constant. Let $h(z)=t$, then $t^{n+d}=1$, we complete the proof of theorem 1 .

Proof of theorem 2. From the conditions of theorem 1, we

$$
\text { know } \frac{f^{n}(z)\left(f^{m}(z)-1\right) \prod_{i=1}^{d} f\left(q_{i} z\right)-1}{g^{n}(z)\left(g^{m}(z)-1\right) \prod_{i=1}^{d} g\left(q_{i} z\right)-1}=c \text {, }
$$

$C$ is nonzero constant, so we rewriting it as

$$
\begin{aligned}
& f^{n}(z)\left(f^{m}(z)-1\right) \prod_{i=1}^{d} f\left(q_{i} z\right)-1+c \\
= & c g^{n}(z)\left(g^{m}(z)-1\right) \prod_{i=1}^{d} g\left(q_{i} z\right)
\end{aligned}
$$

First we let $\quad F=f^{n}(z)\left(f^{m}(z)-1\right) \prod_{i=1}^{d} f\left(q_{i} z\right)$, $G=g^{n}(z)\left(g^{m}(z)-1\right) \prod_{i=1}^{d} g\left(q_{i} z\right)$.

If $c \neq 1$, From (6) and the lemma 1, we have 


$$
\begin{aligned}
T(r, F) & \leq \bar{N}\left(r, \frac{1}{F}\right)+\bar{N}\left(r, \frac{1}{F-1+c}\right) \\
& +S(r, f) \leq(1+m+d) T(r, f) \\
& +\bar{N}\left(r, \frac{1}{G}\right)+S(r, f) \\
& \leq(1+m+d) T(r, f) \\
& +(1+m+d) T(r, g)+S(r, f)
\end{aligned}
$$

From the lemma 5, we know

$$
T(r, F)=(n+m+d) T(r, f)+S_{q}(r, f)
$$

Combining (7) and (8), we have

$$
\begin{aligned}
(n-1) T(r, f) \leq & (1+m+d) T(r, g) \\
& +S_{q}(r, f)+S_{q}(r, g)
\end{aligned}
$$

Applying the same methods of (9),we have

$$
\begin{aligned}
(n-1) T(r, g) \leq(1+ & m+d) T(r, f) \\
& +S_{q}(r, f)+S_{q}(r, g)
\end{aligned}
$$

Combining (9) and (10), we have

$$
\begin{aligned}
& (n-m-d)(T(r, f)+T(r, g)) \\
& \quad \leq S_{q}(r, f)+S_{q}(r, g)
\end{aligned}
$$

Which is a contradicts with $n \geq m+5 d$.

Then $c=1$, from (6), we have

$$
\begin{gathered}
f^{n}(z)\left(f^{m}(z)-1\right) \prod_{i=1}^{d} f\left(q_{i} z\right) \\
=g^{n}(z)\left(g^{m}(z)-1\right) \prod_{i=1}^{d} g\left(q_{i} z\right) \\
\text { Let } h(z)=\frac{f(z)}{g(z)} \text {, then } \\
g^{m}\left(h^{n+m}(z) \prod_{i=1}^{d} h\left(q_{i} z\right)-1\right)=h^{n}(z) \prod_{i=1}^{d} h\left(q_{i} z\right)-1,
\end{gathered}
$$

If $h(z)$ is not a constant, then $h(z)$ is meromorphic.

If 1 is exceptional value of $h^{n+m}(z) \prod_{i=1}^{d} h\left(q_{i} z\right)-1$, Then

$$
\begin{aligned}
& T\left(r, h^{n+m}(z) \prod_{i=1}^{d} h\left(q_{i} z\right)\right) \leq \bar{N}\left(r, h^{n+m}(z) \prod_{i=1}^{d} h\left(q_{i} z\right)\right) \\
& +\bar{N}\left(r, \frac{1}{h^{n+m}(z) \prod_{i=1}^{d} h\left(q_{i} z\right)}\right)+\bar{N}\left(r, \frac{1}{h^{n+m}(z) \prod_{i=1}^{d} h\left(q_{i} z\right)-1}\right) \\
& \leq(2+2 d) T(r, h)+S_{q}(r, h)
\end{aligned}
$$

From (11) ,we have

$$
\begin{gathered}
(n+m) T(r, h(z))=T\left(r, h^{n+m}(z)\right) \leq T\left(r, h^{n+m}(z) \prod_{i=1}^{d} h\left(q_{i} z\right)\right) \\
+T\left(r, \frac{1}{\prod_{i=1}^{d} h\left(q_{i} z\right)}\right)+S_{q}(r, h) \leq(2+3 d) T(r, h)+S_{q}(r, h)
\end{gathered}
$$

Which is a contradicts with $n \geq m+5 d$,

So 1 is not exceptional value of $h^{n+m}(z) \prod_{i=1}^{d} h\left(q_{i} z\right)-1$.

So there exists a point $Z_{0}$ such that

$$
h^{n+m}\left(z_{0}\right) \prod_{i=1}^{d} h\left(q_{i} z_{0}\right)=1
$$

Since $g(z)$ is entire, so $h^{n}\left(z_{0}\right) \prod_{i=1}^{d} h\left(q_{i} z_{0}\right)=1$,

$$
\begin{aligned}
& \text { so } h^{m}\left(z_{0}\right)=1 \text {, then } \\
& T\left(r, h^{n+m}(z) \prod_{i=1}^{d} h\left(q_{i} z\right)\right) \leq \bar{N}\left(r, h^{n+m}(z) \prod_{i=1}^{d} h\left(q_{i} z\right)\right) \\
& +\bar{N}\left(r, \frac{1}{h^{n+m}(z) \prod_{i=1}^{d} h\left(q_{i} z\right)}\right)+\bar{N}\left(r, \frac{1}{h^{m}(z)-1}\right) \\
& \leq(2+2 d+m) T(r, h)+S_{q}(r, h)
\end{aligned}
$$

From (10) we have

$$
\begin{aligned}
& (n+m) T(r, h(z))=T\left(r, h^{n+m}(z)\right) \leq T\left(r, h^{n+m}(z) \prod_{i=1}^{d} h\left(q_{i} z\right)\right) \\
& +T\left(r, \frac{1}{\prod_{i=1}^{d} h\left(q_{i} z\right)}\right)+S_{q}(r, h) \leq(2+3 d+m) T(r, h)+S_{q}(r, h)
\end{aligned}
$$

Which is a contradicts with $n \geq m+5 d$.

So $h(z)$ is a constant. If $h(z)=t$, then $t^{n+d}=t^{m}=1$, we complete the proof of theorem 2 .

\section{ACKNOWLEDGMENT}

The research was supported by the National Natural Science Foundation of China (project no: 11171184), and the Project of Shandong Province Higher Educational Science and Technology Program (project no: J09LA55)

\section{REFERENCES}

[1] Z.X.Chen, Z.B.Huang,X.M.Zheng, On properties of difference polynomials.Acta Math Sci, vol. 31B,pp.627-633,2011.

[2] J.L.Zhang, Value distribution and shared sets of diferences of meromorphic functions. Math Anal Appl, vol 367, pp.401-408,2010.

[3] Z.B.Huang,Z.X.Chen, A Clunie lemma for difference and qdifference polynomials Bull. Aust. Math. Soc. vol81, pp. 23-32, 2010.

[4] J.L.Zhang, R.Korhonen, On the Nevanlinna characteristic of f(qz) and its applications,J.Math. Anal.Appl. vol369, pp.537-544.,2010.

[5] C.C.Yang, I.Laine,On analogies between nonlinear difference and differential equations. Pro Japan Acad Ser A, vol86, pp. 10-14. 2010. 
[6] Y. M. Chiang, S. J. Feng.On the Nevanlinna characteristic of $f(z+\eta)$ anddifference equations in the complex plane. Ramanujian J. vol. 16, pp.105-129,2008.

[7] R. G. Halburd and R. J. Korhonen. Meromorphic solutions of difference equations, integrability and the discrete Painleve equations, J. Phys. A. vol.40, pp.1-38,2007..

[8] C.C.Yang, H.X.Yi. Uniqueness Theory of Meromorphic Functions. Kluwer Academic Publishers, 2003.

[9] I.Laine. Nevanlinna Theory and Complex Differential Equations, Walter de Gruyter, Berlin, 1993.
[10] W.K.Hayman. Meromorphic Functions.Clarendon Press, Oxford. 1964.

[11] D.Barnett,R.G.Halburd,R.J.Korhonen,W.Morgan. Nevanlinna theory for the q-difference operator and meromorphic solutions of qdifferenceequations. Proc. Roy. Soc. Edinburgh Sect. A ,vol.137, pp.457-474,2007.

[12] K.Liu, X.L.Liu, T.B.Cao,Value Distributions and Uniqueness of Difference Polynomials,Advance in Difference Equations, vol.2011,Article ID 234215.12 pages. 